\section{Massive haemorrhagic transformation in cardioembolic stroke: the role of arterial wall trauma and dissection}

\author{
G R de Freitas, A Carruzzo, A Tsiskaridze, J A Lobrinus, J Bogousslavsky
}

Keywords: cerebrovascular diseases; haemorragic transformation; cardioembolic stroke; intracranial dissection

\begin{abstract}
pathogenesis of massive haemor transformation is not well established. Fatal haemorrhagic transformation associated with in situ dissection after acute middle cerebral artery (MCA) occlusion in a patient with atrial fibrillation is reported. An 80 year old woman with atrial fibrillation developed mild hemiparesis and aphasia. Brain CT and MRI at 4 and 5 hours, respectively, of symptom onset showed proximal MCA trunk occlusion with developing striatocapsular infarct and hypoperfusion in the superficial MCA territory. A few hours later, she developed massive bleeding into the ischaemic area and died. Pathological examination showed MCA trunk dissection, surrounded by a subarachnoid clot which communicated with the cerebral haematoma. It is suggested that direct arterial wall trauma as a result of cardioembolic MCA occlusion caused bleeding into the infarct. Secondary in situ dissection may be an overlooked mechanism of haemorrhagic transformation. (F Neurol Neurosurg Psychiatry 2001;70:672-674)
\end{abstract}

Haemorrhagic transformation can be a devastating complication of ischaemic stroke. Moreover, as it is the main complication of thrombolytic treatment, a clear understanding of its pathogenesis is essential. Several mechanisms have been proposed, but several issues gested that vascular rupture might play a part in haemorragic transformation. ${ }^{3}$ We describe a patient with atrial fibrillation who presented a fatal intrainfarct haematoma associated with in situ dissection after acute middle cerebral artery (MCA) occlusion.

\section{Case report}

An 80 year old woman, known to have atrial fibrillation, was treated for arterial hypertension. Six years previously she had needed emergency abdominal surgery for mesenteric ischaemia. She was not currently receiving prophylatic oral anticoagulants. On the morning of admission to hospital, she collapsed in remain unresolved. ${ }^{2}$ We have previously sug-
Department of Neurology, Centre Hospitalier Universitaire Vaudois, Lausanne, Switzerland G R de Freitas A Carruzzo

A Tsiskaridze

J Bogousslavsky

Department of Pathology

J A Lobrinus

Correspondence to: Professor J Bogousslavsky Julien.Bogousslavsky@ chuv.hospvd.ch

Received 24 March 2000 and in revised form

12 September 2000

Accepted 11 October 2000

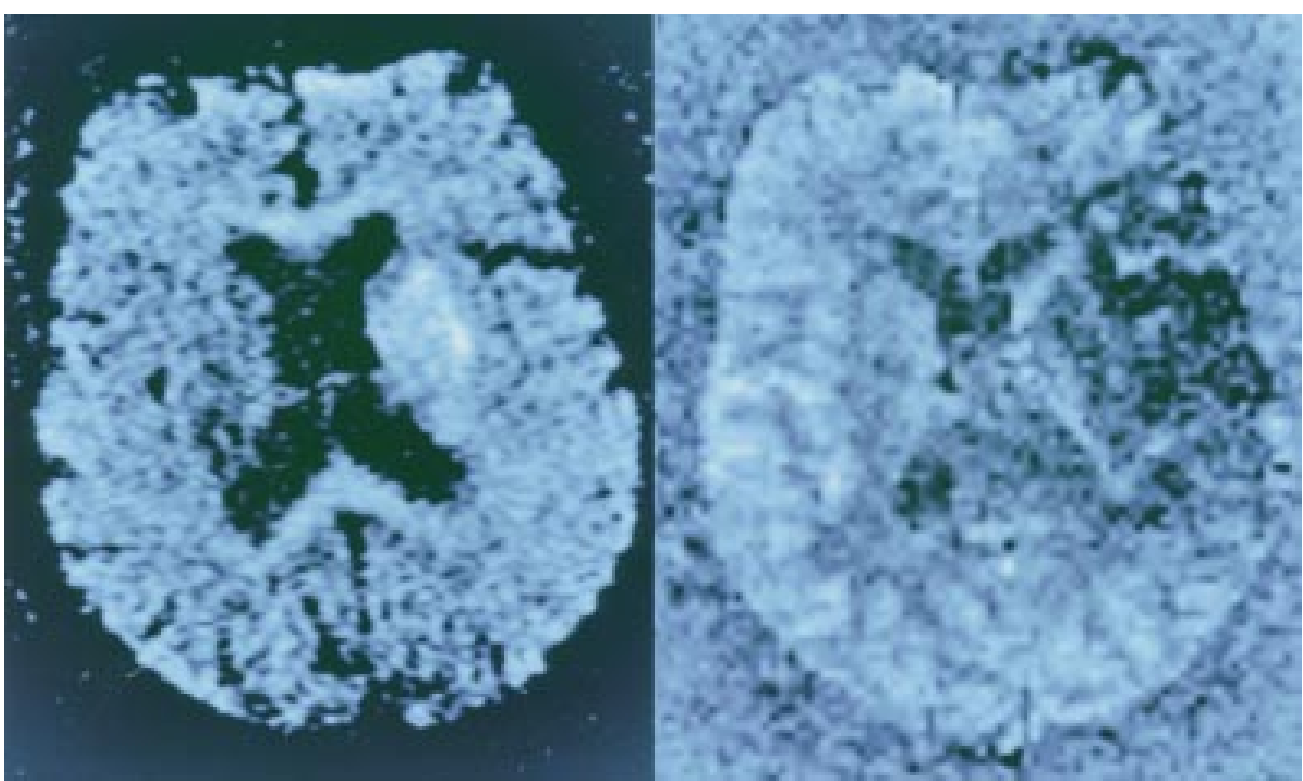

Figure 1 Diffusion weighted (left) and perfusion weighted (right) brain MRI 5 hours after the onset of symptoms. Note (1) the lesion in the left basal ganglia and centrum semiovale and (2) global hypoperfusion in the left middle cerebral artery territory. 

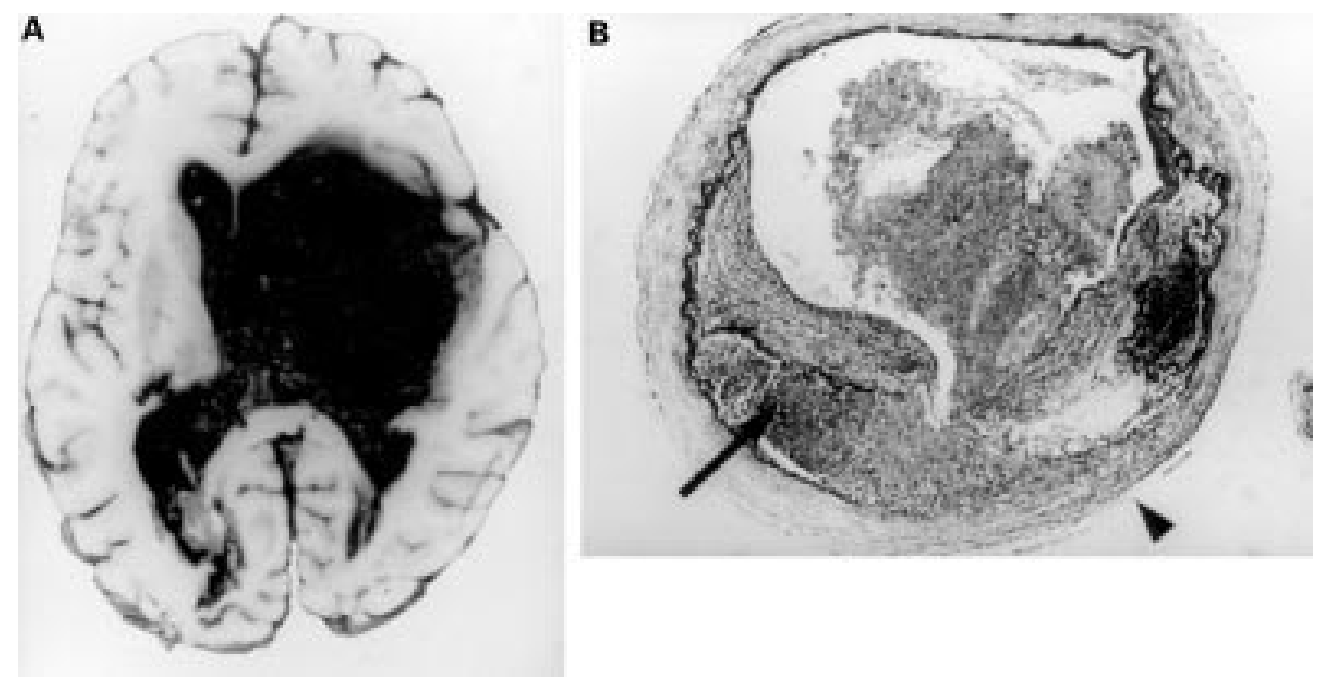

Figure 2 (A) Horizontal brain section disclosing a large haematoma centred in the left basal ganglia, with massive intraventricular haemorrhage. (B) Transverse section of the M1 segment of the left middle cerebral artery (van Gieson-elastin), showing an atheromathous plaque with fresh haemorrhage and dissection between the intimal plaque and the media (arrow), and extension of the blood through the media to the adventitial region (arrowhead). Note the fresh thrombotic material in the lumen.

the bathroom and was found unconscious. Neurological examination in the emergency room showed non-fluent aphasia without spared comprehension, a mild proportional right motor hemisyndrome, and no clouding of consciousness. Two hours later, the paresis evolved to plegia and she presented a right sensory hemisyndrome, a left conjugate ocular deviation, and aphemia. Brain CT, performed 4 hours after the initial symptoms appeared, showed effacement of sulci and MCA trunk occlusion. Diffusion weighted MRI, carried out 1 hour later, showed a lesion in the left basal ganglia and centrum semiovale, and perfusion weighted MRI showed global hypoperfusion in the superficial MCA territory (figure 1). The mean arterial blood pressure was 120 $\mathrm{mm} \mathrm{Hg}$. The patient was given low dose subcutaneous unfractionated heparin (10 000 $\mathrm{IU} /$ day) and oral aspirin (300 mg/day). Sixteen hours later, she became comatose. The CT was repeated and showed a massive left sided intracranial haematoma with intraventricular bleeding and hydrocephalus. The patient died a few hours later.

\section{PATHOLOGICAL EXAMINATION}

Macroscopic brain examination disclosed a 6 $\mathrm{cm}$ diameter haematoma centred in the left basal ganglia, with massive intraventricular haemorrhage (fig $2 \mathrm{~A}$ ), subarachnoid haemorrhage, and brain stem compression with secondary right pontomesencephalic haemorrhage. There was moderate atheromathosis of the proximal segment of the intracranial arteries. On section, the M1 segment of the left MCA was occluded with fresh thrombotic material, and the vessel wall was surrounded by a blood clot which communicated with the intracranial haematoma. No other vessels were filled with thrombotic material. Microscopic examination disclosed confluent zones of necrosis in the superficial territory of the MCA. There were fibrin deposits in the thrombotic material. The MCA lumen was narrowed by an atheromatous plaque. This atheromatous lesion was filled with blood, with dissection of the arterial wall and extension of the blood into the adventitial region. The diagnosis of in situ MCA dissection was established (fig 2 B).

\section{Discussion}

There are two distinct syndromes of haemorragic transformation, ${ }^{1}$ type 1 , a multifocal or pethechial haemorrhagic infarction (haemorrhagic infarction stage $\mathrm{c} 1, \mathrm{c} 2, \mathrm{~d} 1$, and $\mathrm{d} 2$ according to the classification of Moulin $e t a l^{4}$ ), and type 2, a secondary intracranial haematoma that may be identical to primary intraparenchymatous haemorrhage (intrainfarct haematoma stage $\mathrm{c} 1, \mathrm{c} 2, \mathrm{~d} 1$, and $\mathrm{d} 2$ according to the classification of Moulin et $a l^{4}$ ). The pathogenesis of haemorragic transformation is multifactorial, involving reperfusion, vessel wall damage by ischaemia, diapedesis of the intravascular component, collateral circulation, and other factors. ${ }^{12}$ Fisher and Adams suggested that the infarct is caused by blockage of an artery by an embolus, and that subsequent fragmentation of the embolus and relaxation of local vascular spasm makes the embolus move to more distal branches and exposes necrotic tissues and damaged capillaries to reperfusion, resulting in haemorrhage. ${ }^{5}$ Haemorragic transformation has been reported in up to $71 \%$ of cardioembolic infarcts, ${ }^{6}$ and up to $95 \%$ of haemorrhagic infarcts are of cardiac origin. ${ }^{5}$ Our patient had a history of hypertension and atrial fibrillation and had undergone surgery for mesenteric ischaemia 6 years before admission. Embolism is responsible for about $50 \%$ of episodes of mesenteric ischaemia, and the embolus usually originates from a left atrial or ventricular mural thrombus. ${ }^{7}$ However, anticoagulation therapy had not been prescribed. Pathological examination disclosed that a transmural dissection through an atherosclerotic plaque in the proximal part of the MCA was the direct cause of the intracranial 
haematoma. We speculate that direct trauma in an atheromatous vessel due to a cardiac embolus led to intima rupture. This hypothesis is supported by one study in which arterial rupture of the vessel wall caused by a cardiac embolus was reported in two patients, one of whom presented a large fatal intracranial haematoma. ${ }^{8}$ Moreover, this assumption is consistent with animal studies carried out in 1937 by Villaret et al, who suggested that direct stimulation of smooth muscle cells would provoke a local and violent spasm, leading to arterial rupture. ${ }^{9}$ Anatomical studies performed by Globus and Epstein suggested that vessel rupture is the mechanism underlying massive intracranial haematoma; they reported that "vessels were encountered, showing more advanced disintegration, and often displaying a gap through which blood apparently made its escape into the surrounding space" and "vessels were encountered with walls separated (dissected) into several layers by collections of blood." 10 We have also found other reported cases of intracranial dissection followed by type 2 haemorragic transformation, ${ }^{11-13}$ some of which had large intracranial haematomas and ventricular involvement, leading to brain herniation. ${ }^{12}{ }^{13}$ Given the inaccuracy of angiography in the detection of intracranial dissections $^{14}$ and the lack of large pathological studies of large haemorragic transformation (type 2$),{ }^{1}$ we propose that dissection of intracranial arteries may underlie type 2 haemorragic transformation in some patients. We question whether reperfusion is essential for the development of the transformation, as pathological examination of our patient clearly showed occlusion of a MCA by a blood clot. Although our assumption is not supported by experimental studies using animals, such models do not mimic the human condition. ${ }^{15}$ Some species present bleeding after vessel occlusion, whereas others do not. In addition, young, healthy animals are used for modelling stroke, whereas human stroke often occurs in elderly patients with concomitant medical problems. Both our findings and pathological ${ }^{8}$ and experimental ${ }^{9}$ studies also suggest that trauma caused by an embolus could lead to arterial vessel damage and subsequent dissection. Whether intracranial arterial dissection is an overlooked mechanism of type 2 haemorragic transformation still remains uncertain, and pathological studies are still necessary. If this proves to be the case, the implications for the therapeutic use of anticoagulant and thrombolytic drugs in cardioembolic stroke are unknown, but might be determined by clinical studies.

This work was supported in part by a European Neurological Society grant to GRdeF.

1 Hart RG, Easton JD. Hemorrhagic infarcts. Stroke 1986;17:586-9.

2 Lyden PD, Zivin JA. Hemorragic transformation after cerebral ischemia: mechanisms and incidence. Cerebrovasc Brain Metab Rev 1993;5:1-16.

3 Bogousslavsky J, Regli F, Uske A, et al. Early spontaneous hematoma and cerebral infarct: is primary cerebral hemorrhage overdiagnosed? Neurology 1991;41:837-40.

4 Moulin T, Crépin-Leblond T, Chopard JL, et al. Hemorrhagic infarcts. Eur Neurol 1993;34:64-77.

5 Fisher CM, Adams RD. Observations on brain embolism with special reference to the mechanism of hemorrhagic infarction. F Neuropathol Exp Neurol 1951;10:92-3.

6 Jörgensen L, Torvik A. Ischaemic cerebrovascular disease in an autopsy series: Part prevalence, location, pathogenesis and clinical course of cerebral infarcts. $\mathcal{F}$ Neurol Sci 1969;9: 285-320.

7 Kaleya RN, Boley SJ. Acute mesenteric ischemia. Crit Care Clin 1995;11:479-512.

8 Brucher JM, De Smet Y, Gonsette RE. Rupture of the arterial wall in two cases of recent cerebral embolism: morphoogical expression of cerebral vasospasm? I Neurol 1986; 233: $267-70$.

9 Villaret M, Cachera R, Fauvert R. Spasmes des artères cérébrales provoquées par l'embolie expérimentale du cerveau. C R Soc Biol (Paris) 1937:58-61.

10 Globus JH, Epstein JA. Massive cerebral hemorrhage: spontaneous and experimental induced. 7 Neuropathol Exp Neurol 1953;12:107-31.

11 Hochberg FH, Bean C, Fisher CM, et al. Stroke in a 15 year old girl secondary to terminal carotid dissection. Neurology 1975;25:725-9.

12 Nass R, Hays A, Chutorian A. Intracranial dissecting aneurysms in the childhood. Stroke 1982;13:204-7.

13 Schmitt HP, Miltner E. Dissection of the anterior and middle cerebral artery with fatal ischemia following kicks to the head. Forensic Sci Int 1991;49:113-20.

14 Kunze ST, Schiefer W. Angiographic demonstration of dissecting aneurysm of the middle cerebral artery. Neuroradiology 1971;124:512-25.

15 Millikan C. Animal stroke models. Stroke 1992; 23:795-7. 